\title{
High resolution spectroscopy of circumstellar material around A stars ${ }^{\star}$
}

\author{
M. Hempel and J. H. M. M. Schmitt
}

\author{
Hamburger Sternwarte, Gojenbergsweg 112, 21029 Hamburg, Germany
}

Received 23 December 2002 / Accepted 13 June 2003

\begin{abstract}
We have analysed a time series of high resolution spectra $(R=217000)$ of the CaII $\mathrm{K}$ line of 9 stars which are candidates for the presence of circumstellar material from our previous studies. We have searched for variable narrow absorption components similar to those extensively studied in the case of $\beta$ Pictoris. Our data show long-term variations in the spectra of HR 2550 and HR 3685 which can be attributed to the dynamics of circumstellar gas. About one third of the sample stars show variable line absorption but only $\beta$ Pictoris seems to exhibit uniquely strong variations on short (nightly) timescales. In order to examine possible interstellar contributions we compared our results with interstellar data from the literature. The column densities of our absorption features are up to three orders of magnitude higher than those found for the Local Interstellar Cloud.
\end{abstract}

Key words. stars: circumstellar matter - ISM: atoms - stars: individual: $\beta$ Pictoris

\section{Introduction}

Observations with IRAS showed that about $20 \%$ of the nearby A stars are surrounded by dust disks (Cheng et al. 1992). The most prominent prototypes of this class of dusty A type stars are Vega, $\beta$ Pictoris, and $\alpha$ PsA. Such disks have generated particular interest in the framework of young planetary systems. Infrared (IR) observations are sensitive only to dust but not to gas. How can one analyse the gas in such disks? A common method for the detection of gas is to search for molecules like $\mathrm{H}_{2}$ and $\mathrm{CO}$ (Thi et al. 2001). Lecavelier des Etangs et al. (2001) did not detect $\mathrm{H}_{2}$ in the disk of $\beta$ Pictoris. Furthermore, Richter et al. (2002) have searched for $\mathrm{H}_{2}$ emission from six protoplanetary disks, among them three with ISO detections (Thi et al. 2001). Direct detection of molecular hydrogen is difficult because $\mathrm{H}_{2}$ has no dipole moment (Bary et al. 2002). In contrast to Thi et al. (2001), Richter et al. (2002) did not find any convincing $\mathrm{H}_{2}$ emission with TEXES (Lacy et al. 2002) around these objects. Observations of $\mathrm{CO}$ around A-type stars pose problems as well. As shown by Kamp \& Bertoldi (2000), the radiation field of A stars with an effective temperature comparable to Vega $\left(T_{\text {eff, Vega }}=9500 \mathrm{~K}\right)$ is capable of destroying $\mathrm{CO}$ in the circumstellar environment. Alternatively, the presence of gas can be diagnosed by circumstellar (CS) absorption features in CaII K. The CaII K line is easily accessible from high sensitivity ground-based observations (Hobbs et al. 1985). Unfortunately, such absorption features could in

Send offprint requests to: $\mathrm{M}$. Hempel,

e-mail: mhempel@hs.uni-hamburg.de

* Based on observations collected at the European Southern Observatory, La Silla, Chile. principle also be due to interstellar (IS) absorptions produced by clouds along the line of sight. However, the observed variability of the absorption features, at least in the prototypical case of $\beta$ Pictoris, definitely proves a CS origin. The clear dependence of the absorptions on the rotational properties, their preference for stars with low gravities (Holweger \& Rentzsch-Holm 1995, hereafter HRH, Holweger, et al. 1999, hereafter HHK), and the IR emission in some cases strongly suggest their circumstellar origin.

Several surveys have been carried out in order to find circumstellar absorptions (Lagrange-Henri et al. 1990; Lecavelier des Etangs et al. 1997c; Welsh et al. 1998). HRH \& HHK carried out a systematic program to search for the presence of gas in the circumstellar environment. In the course of these studies we searched for circumstellar CaII $\mathrm{K}$ lines in A stars on the southern hemisphere and determined $\mathrm{Ca}$ abundances in order to search for accretion-induced abundance effects in a sample of dusty and dust-free A stars taken mainly from the Cheng et al. (1992) list. The results show that about $30 \%$ of the A stars studied have narrow absorption features.

In the prototypical case of $\beta$ Pictoris the CS components are a well-studied phenomenon (Vidal-Madjar et al. 1986, 1994; Lagrange et al. 1992, 1995; Beust et al. 1998; Petterson \& Tobin 1999). The star shows a seemingly stable central absorption and a variable component which may as well vanish at times (Lagrange et al. 1996; Beust et al. 1998; Petterson \& Tobin 1999). Observations of variable CS features are explained in terms of the so-called falling evaporating bodies (FEB) scenario (Ferlet et al. 1987; Beust et al. 1990, 1991; Thébault \& Beust 2001): When kilometre-sized cometary-like objects on star-grazing orbits pass near $\beta$ Pictoris, material is 
evaporated leading to imprints in the photospheric spectrum. Detailed analysis of these features allow estimates on both the column densities and occurrence rates of the absorbing clouds and thus provide insights in the stages of early stellar evolution connected with the formation of planetary systems (Lagrange-Henri et al. 1992; Ferlet et al. 1993; Lagrange et al. 1996; Beust \& Morbidelli 1996, 2000; Thébault \& Beust 2001).

Concerning variability of narrow CaII $\mathrm{K}$ absorptions from stars of the Cheng et al. (1992) compilation only $\beta$ Pictoris has been studied in a detailed long-term study (Petterson \& Tobin 1999) so far. For two other stars not included in the Cheng et al. (1992) sample, HR 10 and HD 85905, Welsh et al. (1998) have found suchlike variations from their analysis of CaII $\mathrm{K}$ and NaI D absorptions as well.

Taking the FEB scenario as a working hypothesis, one expects the FEB scenario also to be applicable to the other stars in our sample. The goal of this work is to search for possible variabiliy in CaII $\mathrm{K}$ originating in the circumstellar environment.

In Sect. 2 we describe our observations as well as data reduction and analysis. In Sect. 3 our results are presented with emphasis on the individual stars. In Sect. 4 we discuss to what extent the observed absorption features are indicative of IS or CS absorptions. Finally, in Sect. 5 we summarize our conclusions.

\section{Observations and spectral analysis}

Our stellar sample listed in Table 1 consists of all stars on which Cheng et al. (1992) have based their search for cirumstellar dust which were accessible during our observing nights and known to exhibit narrow absorption features in the core of the CaII K line. We have added the $\lambda$ Bootis star HR 4881 to our program stars since it is known to possess a prominent narrow absorption component which can be attributed to the presence of circumstellar gas (HHK). In Table 1 we compiled the basic stellar parameters as well as the galactic coodinates of our sample stars. Concerning the presence of CS dust, IRAS data for our stellar sample are discussed in Cheng et al. (1992), Oudmaijer et al. (1992), and Jura (1991) (only for HR 4796). For a discussion on the ages of the stars see HHK, Sects. 5 and 6.

Observations have been carried out with the ESO 3.6-m telescope in two consecutive nights (2002 March 25/26). The telescope was equipped with the CES system at a resolving power of 217000 . The exposure lengths were chosen to guarantee a $S / N$ above 200. We observed the CaII K line of each of our program stars several times each night (see Table 2 ) in order to search for short-term variability. As in our previous work (see Kamp et al. 2002 and references therein) we have reduced our spectra with MIDAS routines. We used Th - Ar spectra for wavelength calibration. The fundamental parameters $T_{\text {eff }}$ and $\log g$ as well as $v \sin i$ were taken from HHK.

In order to distinguish the stellar profile from a narrow absorption component we calculated synthetic spectra based on ATLAS9 models (Kurucz 1992) with our line formation code LINFOR (HRH). For further details concerning this procedure see HHK (spectra are shown in Fig. 1) and references therein. The line data was taken from the VALD database (Kupka et al. 1999). We measured the equivalent width $W_{\mathrm{K}}$ of the narrow absorption features with respect to the photospheric line core. Furthermore, we determined the radial velocities with respect to the star and - for comparison with interstellar data - their heliocentric velocities using appropriate corrections.

\section{Results}

In Table 2 we present the results of our analysis of the CaII K lines for our program stars. For comparison, we included data from our previous work obtained with the CAT/CES-system at a resolution of $R \approx 70000$ (HHK) in order to search for long-term variability. We specifically list the dates and times for all obtained spectra, the equivalent widths, both radial velocities $v_{\mathrm{K}}$ of the narrow absorption features with respect to the star and (for the 2002 data) heliocentric corrected velocities $v_{\text {hel }}$. Since heliocentric velocities were not available for the 1996 data, follow-up observations are desirable to easily distinguish CS from IS origin of the features for binaries. From the measured equivalent widths $W_{\mathrm{K}}$ of the narrow absorptions we calculated the column densities $\log N$ according to LagrangeHenri et al. (1990). Each star is shown in Figs. 1-9 to illustrate the quality of our data.

All program stars have previously been observed. Although the former spectral resolution of $R=70000$ was not as high as it is in the current work we are able to determine differences in both equivalent width and radial velocity of the narrow CaII $\mathrm{K}$ absorptions.

\subsection{The archetype of spectroscopic variations: HR 2020 ( $\beta$ Pic)}

$\beta$ Pictoris is the prototype of dusty A type stars with variable circumstellar components. Since the successful imaging of its edge-on circumstellar disk using coronographic techniques (Smith \& Terrile 1984) this star has been extensively studied. The variability in the core of the CaII $\mathrm{K}$ line on short timescales is attributed to falling evaporating bodies (FEB's) (Thébault \& Beust 2001 and references therein) - cometary-like objects which produce the absorbing clouds as they approach the star. Petterson \& Tobin (1999) have carried out a long-term study on the spectral variability of $\beta$ Pictoris in $\mathrm{Ca} \mathrm{H}$ and $\mathrm{K}$ between 1994 and 1996. They analysed 313 spectra and found that typically 4-5 clouds are in the line of sight. Event rates of 400 per year for high velocity features (HVF's) are reported whereas low velocity features are seemingly more stable and may even persist for several nights. They conclude that their results are consistent with the FEB scenario. The line cores of our new spectra of HR 2020 are - as expected - conspicuously different from our data obtained in 1996. An example is given in Fig. 1. In the first night (2002 Mar. 25/26) we have detected three absorption components in the core of the CaII $\mathrm{K}$ line whereas in the second night (2002 Mar. 26/27) a fourth broad redshifted feature with high radial velocity $\left(v_{\mathrm{K}} \approx 100 \mathrm{~km} \mathrm{~s}^{-1}\right.$, $W_{\mathrm{K}} \approx 9 \mathrm{m \AA}$ ) with respect to the star occurred (see Fig. 1). We note that this HVF is present in all our spectra of HR 2020 taken in the second night and absent in all spectra recorded in the first night. Thus we interpret this as an unambiguous detection. As can be seen from Fig. 1 (bottom) this feature 
Table 1. Our program stars. Visual magnitudes, spectral types, as well as galactic coordinates were take from the Bright Star Catalogue (Hoffleit \& Warren 1991). Distances have been calculated from HIPPARCOS parallaxes (ESA 1997). Stellar parameters have been taken from HHK. The last column denotes whether a star is known to possess CS dust ( ${ }^{a}$ Cheng et al. 1992, ${ }^{b}$ Jura 1991).

\begin{tabular}{|c|c|c|c|c|c|c|c|c|c|c|c|}
\hline HR & Name & HD & $m_{v}$ & $\begin{array}{c}\text { Spectral } \\
\text { Type }\end{array}$ & $\begin{array}{c}d \\
(\mathrm{pc})\end{array}$ & $l$ & $b$ & $\begin{array}{l}T_{\text {eff }} \\
(\mathrm{K})\end{array}$ & $\begin{array}{c}\log g \\
\left(\mathrm{~cm} \mathrm{~s}^{-2}\right)\end{array}$ & $\begin{array}{r}v \sin i \\
\left(\mathrm{~km} \mathrm{~s}^{-1}\right)\end{array}$ & dusty? \\
\hline 2020 & $\beta$ Pic & 39060 & 3.85 & A5 V & 19.3 & 258.36 & -30.61 & 8200 & 4.24 & 132 & $\operatorname{yes}^{a}$ \\
\hline 2550 & $\alpha$ Pic & 50241 & 3.27 & A7 IV & 30.3 & 271.92 & -24.10 & 7530 & 3.48 & 205 & no $^{a}$ \\
\hline 3485 & $\delta \mathrm{Vel}$ & 74956 & 1.96 & A1 V & 24.5 & 272.08 & -24.10 & 9250 & 3.79 & 178 & $\mathrm{no}^{a}$ \\
\hline 3685 & $\beta \mathrm{Car}$ & 80007 & 1.68 & A2 IV & 34.1 & 285.98 & -14.41 & 9090 & 3.08 & 133 & no $^{a}$ \\
\hline 4796 & & 109573 & 5.80 & A0 & 67.1 & 299.72 & +22.91 & 10270 & 4.45 & 150 & yes $^{b}$ \\
\hline 4881 & & 111786 & 6.15 & A0 III & 60.2 & 303.08 & +36.13 & 7440 & 3.96 & 126 & - \\
\hline 5367 & $\psi \mathrm{Cen}$ & 125473 & 4.05 & A0 IV & 75.8 & 321.72 & +21.71 & 10250 & 3.71 & 132 & $\operatorname{yes}^{a}$ \\
\hline 6519 & $51 \mathrm{Oph}$ & 158643 & 4.81 & $\mathrm{~B} 9.5 \mathrm{Ve}$ & 130.7 & 2.52 & +5.34 & 9930 & 3.09 & 272 & $\operatorname{yes}^{a}$ \\
\hline 6556 & $\alpha \mathrm{Oph}$ & 159561 & 2.08 & A5 III & 14.3 & 35.89 & +22.57 & 7960 & 3.62 & 210 & no $^{a}$ \\
\hline
\end{tabular}
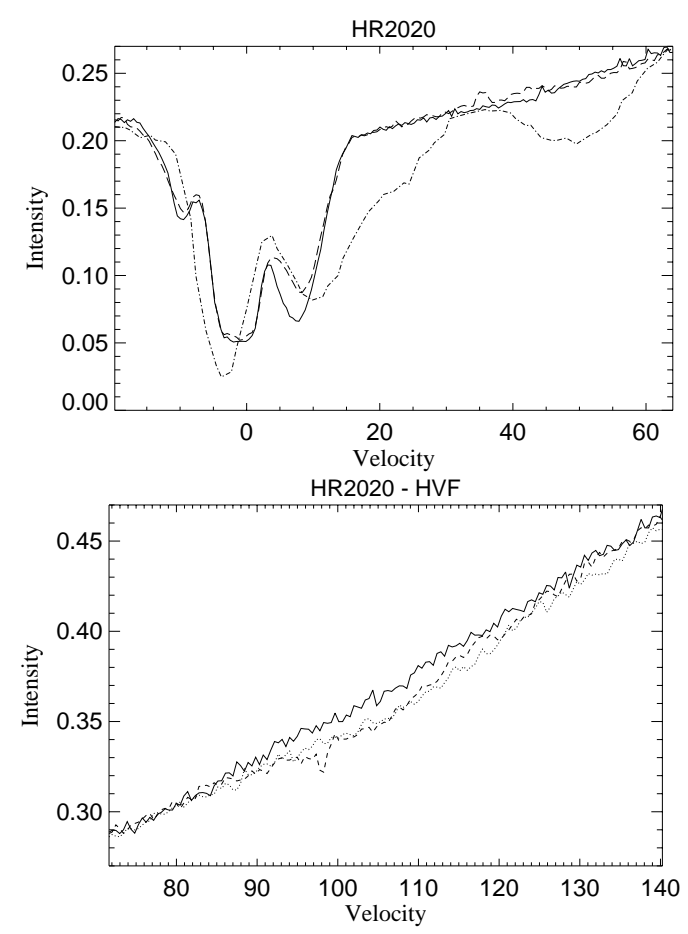

Fig. 1. Upper: HR 2020, obtained on 2002 Mar. 25, UT 23:37 (solid line), on 2002 Mar. 26, UT 23:25 (dashed line), and on 1996 Feb. 29, UT 00:11 (dashed-dotted line). Note the nightly variation at $\sim 8 \mathrm{~km} \mathrm{~s}^{-1}$ in the 2002 spectra. Lower: redward line wing of HR 2020 on 2002 Mar. 25, UT 23:37 (solid line). For comparison, the high velocity feature detected in the second night is shown. Dashed line: 2002 Mar. 26, UT 23:25, dotted line: 2002 Mar. 27, UT 01:19. The radial velocity with respect to the star of this feature corresponds to $v_{\mathrm{K}} \approx 100 \mathrm{~km} \mathrm{~s}^{-1}$ and $v_{\mathrm{K}} \approx 110 \mathrm{~km} \mathrm{~s}^{-1}$, respectively.

shows a slight speedup from $100 \mathrm{~km} \mathrm{~s}^{-1}$ to $110 \mathrm{~km} \mathrm{~s}^{-1}$ during the night. Similar observations have been made by Petterson \& Tobin (1999). They report the observation of a persistent HVF decreasing in redshift on 1995 September 10. Furthermore, our spectra show that the depth of the redshifted component with $v_{\mathrm{K}} \approx 8 \mathrm{~km} \mathrm{~s}^{-1}$ weakened in the second night. Since our data show both clear nightly variations as well as HVFs we confirm the results given by Petterson \& Tobin (1999).

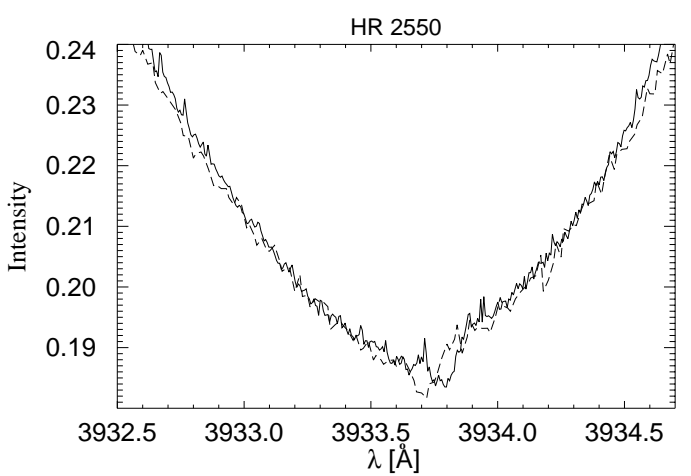

Fig. 2. HR 2550 recorded on 2002 Mar. 26, UT 00:33 (solid line) and 1996 Feb. 29, UT 01:24 (dashed line). Note the change of the radial velocity of the absorption feature in the line core.

\subsection{Positive result: HR 2550 and HR 3685}

\subsubsection{HR 2550 ( $\alpha$ Pic)}

HR 2550 (see Fig. 2) shows a weak absorption feature which slightly changes its radial velocity within the 6 years between our observations. This suggests that the feature is produced by CS gas moving towards the star. We note that this detection of variability in Ca II K is based on data with significantly different spectral resolutions. However, the equivalent width of this feature does not seem to vary. Interestingly, HR 2550 (= GJ 248) is a X-ray source (Hünsch et al. 1999). This is not expected for A-type stars. Therefore it is possible that the Xray emission arises from an unresolved late-type companion. This scenario allows for the possibility that the absorption is of IS origin and the change of radial velocity may be due to the binarity. We note that a significant contribution of the Local Interstellar Cloud (LIC) and the G Cloud (Redfield \& Linsky 2002, 2000; Lallement \& Bertin 1992 ${ }^{1}$ ) can be ruled out (cf. Table 3). From our present knowledge we cannot finally assess the nature of the narrow absorption. Thus HR 2550 clearly deserves further study.

1 S. Redfield, Colorado Model of the Local Interstellar Cloud,

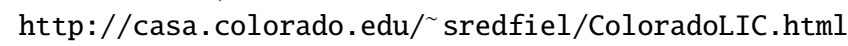




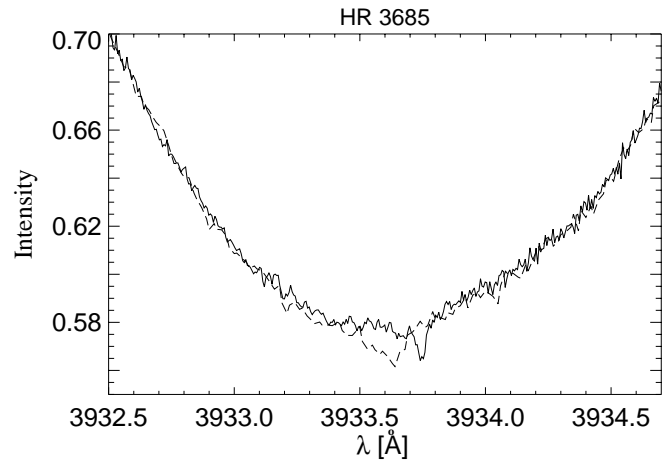

Fig. 3. The Ca II K line of HR 3685 recorded on 2002 Mar. 26, UT 00:13 (solid line) and 1996 Feb. 29, UT 03:30 (dashed line). The feature changed in both radial velocity and shape.

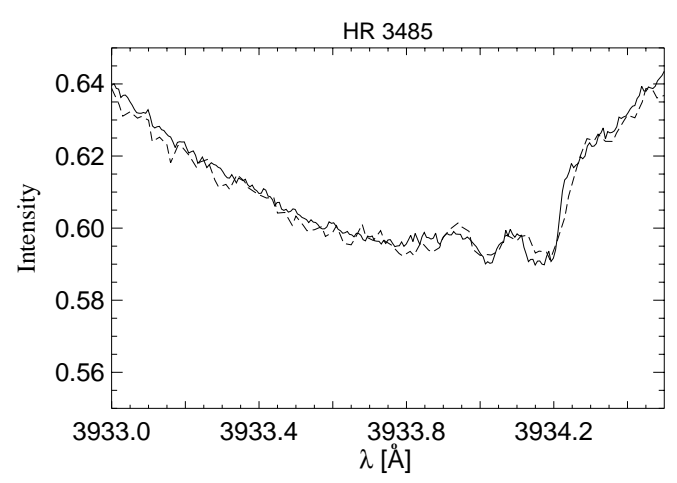

Fig. 4. Spectra of HR 3485 obtained on 2002 Mar. 26, UT 00:07 (solid line) and 1996 Feb. 28, UT 05:09 (dashed line). The features do not show any long-term variability.

\subsubsection{HR 3685 ( $\beta$ Car)}

HR 3685 shows variations in both $W_{\mathrm{K}}$ and $v_{\mathrm{K}}$ (see Fig. 3). Furthermore, despite the different spectral resolution of our datasets, a change of the shape of the feature is discernible. The data reveals that the feature which was detectable in February 1996 in the core of the Ca II K line is now slightly redshifted, indicating CS gas moving towards the star. We are not aware of the presence of companion to HR 3685. We note that the ROSAT-PSPC countrate is below $0.0004 \mathrm{cts} / \mathrm{s}$ (Berghöfer et al. 1999) which does not suggest the existence of a late-type companion. From our present knowledge we have to conclude that the narrow absorptions are due to CS origin.

\subsection{Negative result: No variation and binarities}

\subsubsection{HR 3485 ( $\delta$ Vel)}

HR 3485 has two redshifted absorbing components which did not change in both equivalent width and velocity as visible in Fig. 4. This suggests that these features may be of interstellar origin as well. We will discuss difficulties encountering concerning the distinction between CS and IS origin in the next section.

\subsubsection{HR 4796}

HR 4796 is a star in a binary system with circumstellar material similar to $\beta$ Pictoris. Coronographic images show a spatially resolved ring-like structure of circumstellar dust (Schneider et al. 1999). The ring itself lies $\sim 70 \mathrm{AU}$ from the central star, is about $15 \mathrm{AU}$ thick and has an inclination of $13^{\circ} \pm 1^{\circ}$ with respect to the line of sight (Telesco et al. 2000 and references therein). Therefore HR 4796 appears to be a good candidate for variable circumstellar absorption and possible falling evaporating bodies. Our spectra do not show any hints of variability (Fig. 5). Although our spectra of HR 4796 are unfortunately rather noisy variable CS components should have shown up in our data if present. The reason for the non-detection may be that either the disk of HR 4796 is devoid of gas or at least much thinner than that of $\beta$ Pictoris - Telesco et al. (2000) found from images at $10 \mu \mathrm{m}$ and $18 \mu \mathrm{m}$ that the inner hole of the HR 4796 disk is relatively empty - or that the inclination of HR 4796 is too low: the images of HR 4796 show that we do not observe the circumstellar disk edge-on as it is the case for $\beta$ Pictoris but rather at a much lower inclination.

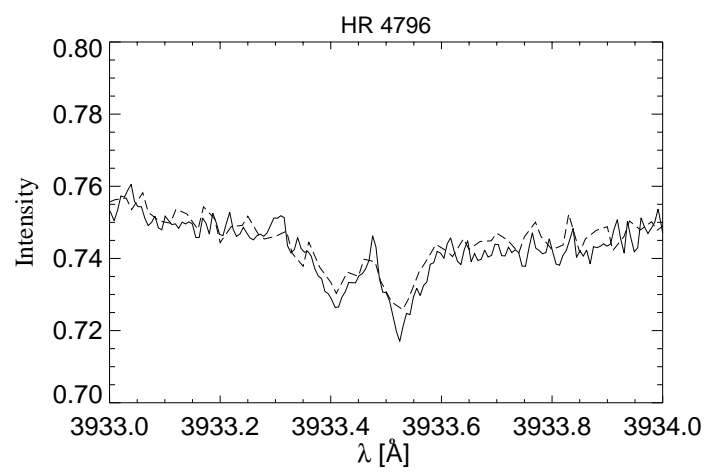

Fig. 5. HR 4796 obtained on 2002 Mar. 26, UT 02:44 (solid line) and 1996 Feb. 29, UT 03:43 (dashed line). Despite the presence of circumstellar dust no signs of variable gas motions are detectable.

\subsubsection{HR 4881}

HR 4881 is the only star in our sample not taken from the Cheng et al. (1992) list. We included it because detailed abundance studies of HR 4881 (Stürenburg 1993) showed a metaldeficiency resembling the surface composition of the $\lambda$ Bootis stars. The members of this small subgroup of A stars show solar abundances of lighter elements like $\mathrm{C}, \mathrm{N}$, and $\mathrm{O}$ whereas heavier elements are underabundant. The metal deficiency of the $\lambda$ Bootis stars is attributed to accretion of depleted gas after separation of gas and dust (Venn \& Lambert 1990) either from their circumstellar environment or from interaction with diffuse interstellar clouds (Kamp \& Paunzen 2002). We observed HR 4881 in previous observing runs and detected a conspicuous narrow absorption feature near the core. The overall shape of this feature remains unchanged for years whereas the radial velocity with respect to the star changes. This is illustrated in Fig. 6. This change (not its origin as already outlined in HHK) is due to the presence of an unresolved companion 


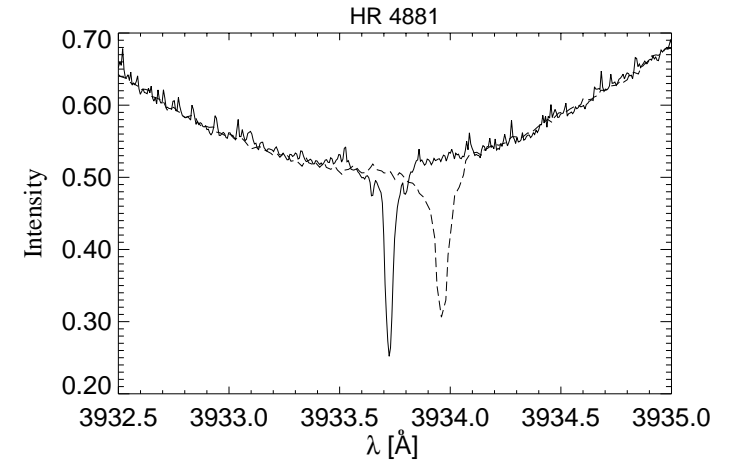

Fig. 6. The $\lambda$ Bootis star HR 4881 obtained on 2002 Mar. 26, UT 03:50; (solid line) and 1996 Feb. 28, UT 07:40 (dashed line). The change of the radial velocity of the narrow absorption feature can be ascribed to the binarity of HR 4881 .

of early spectral type F. We note that we find a small difference of the measured equivalent widths between our different datasets. Concerning the distance to the edge of the LIC of only $0.05 \mathrm{pc}$ it is unlikely that this absorption originates in the LIC but is due to one or more interstellar clouds or circumstellar gas. Despite our spectral resolution of $R=217000$ only one narrow absorption shows up. If this feature thus arises from more than one IS clouds, they must have nearly identical radial velocities within a range of $\sim 5 \mathrm{~km} \mathrm{~s}^{-1}$. In case the feature originates from one single cloud, we note for comparison, that the maximum dimension of the LIC is $6.8 \mathrm{pc}$, corresponding to $\log N\left(\mathrm{Ca}^{+}\right)=10.68$ (Redfield \& Linsky 2000). Despite the fact that the change in radial velocity is due to the binarity of HR 4881, recalling the $\lambda$ Bootis nature of HR 4881 we cannot rule out a CS origin of the narrow absorption feature. Taking the differences of the radial velocities of the absorption features from Table 2 we can estimate very crudely that the distance of both stellar components is $\sim 2 \mathrm{AU}$. Since this is well below the typical diameter of circumstellar disks this finding is consistent with the assumption that both stars are embedded in circumstellar gas.

\subsubsection{HR 5367 ( $\psi$ Cen)}

HR 5367 (Fig. 7) shows a more complex absorption feature in comparison to our 1996 data. Instead of one narrow absorption

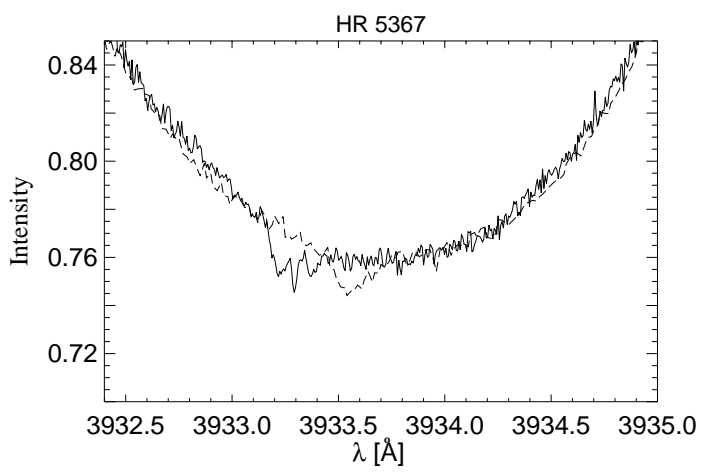

Fig. 7. HR 5367 on 2002 Mar. 26, UT 06:24 (solid line) and 1997 Sep. 15, UT 23:57 (dashed line). component we found three of them in our higher resolution data. Although they show radial velocity variations, similar to HR 4881, the shape of these features does not change. We attribute this to the binarity of HR 5367.

\subsubsection{HR 6519 (51 Oph)}

Waters et al. (1988) first noticed the presence of circumstellar dust around HR 6519. CS gas was suspected by LagrangeHenri et al. (1990) and proven by UV observations (Grady \& Silvis 1993) which show time-variable, redshifted absorptions similar to those studied extensively in the case of $\beta$ Pictoris. This led Crawford et al. (1997) to observe the CaII K line of HR 6519 at much higher resolution $(R \sim 860000)$ using the Ultra-High-Resolution Facility (UHRF) at the AngloAustralian Telescope. They state the detection of five absorption components. In order to search for possible variability they degraded their observations to a resolving power of $R=$ 100000 (i.e., half the resolution of our spectra presented in this paper), enabling them to compare them to data obtained by Lagrange-Henri et al. (1990). The analysis of Crawford et al. (1997) does not show evidence for spectral variability in CaII K, while Roberge et al. (2002) report the detection of timevariable NI, NII, OI, and FeIII lines from FUSE observations in their recent study of HR 6519. Moreover, HR 6519 shows clear evidence for the presence of circumstellar gas from the detection of the FeII line at $4583.84 \AA$ (Dunkin et al. 1997) which is not observed in the interstellar medium since it arises from a metastable level $2.8 \mathrm{eV}$ above the ground state. Lecavelier et al. (1997a, 1997b) have detected CI as well as absorption from MgII and excited levels of FeII originating in the CS environment. Thus the presence of circumstellar dust and gas around 51 Oph is clearly established. In CaII K, HR 6519 (Fig. 8) exhibits a conspicuous narrow absorption which even at low resolution appears to consist of more than one component. In our high resolution 2002 data four components are clearly distinguishable - maybe even more are present. Despite the complexity of the absorption no nightly variations are visible. Even when comparing the spectra with our older data, we do not find a clear evidence for long-term variability but we cannot rule out possible variations.

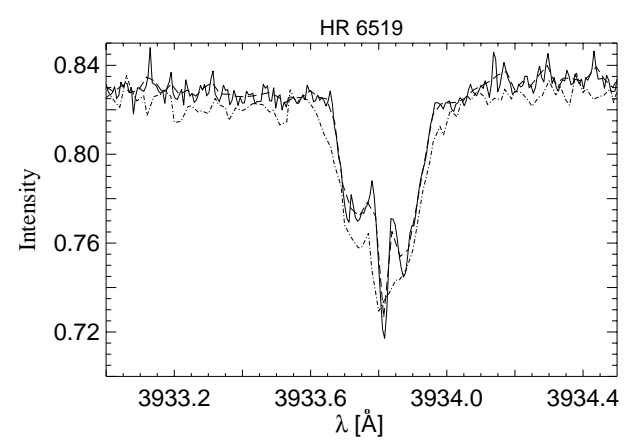

Fig. 8. HR 6519 on 2002 Mar. 26, UT 08:20 (solid line) and 1996 Feb. 29, UT 09:15 (dashed-dotted line). The advantage of the higher spectral resolution is obvious. For comparison, the dashed line shows the 2002 spectrum degraded to the resolution of our 1996 data. 
Table 2. Results of our analysis. See Sect. 2 for details.

\begin{tabular}{|c|c|c|c|c|c|c|}
\hline HR & Date & Time (UT) & $\begin{array}{c}W_{\mathrm{K}} \\
(\mathrm{m} \AA)\end{array}$ & $\begin{array}{r}v_{\mathrm{K}} \\
\left(\mathrm{km} \mathrm{s}^{-1}\right)\end{array}$ & $\begin{array}{r}v_{\text {hel }} \\
\left(\mathrm{km} \mathrm{s}^{-1}\right)\end{array}$ & $\begin{array}{l}\log N \\
\mathrm{~cm}^{-2}\end{array}$ \\
\hline 2020 & 2002 Mar. 25 & $23: 37$ & 173 & $-10,0,8$ & $12,22,30$ & 12.26 \\
\hline 2020 & 2002 Mar. 26 & $00: 20$ & 173 & $-10,0,8$ & $12,22,30$ & 12.26 \\
\hline 2020 & 2002 Mar. 26 & $01: 31$ & 169 & $-10,0,8$ & $12,22,30$ & 12.25 \\
\hline 2020 & 2002 Mar. 26 & 01:42 & 169 & $-10,0,8$ & $12,22,30$ & 12.25 \\
\hline 2020 & 2002 Mar. 26 & $23: 25$ & $162 / 9$ & $-10,0,8 / 100$ & $12,22,30 / 122$ & 12.24 \\
\hline 2020 & 2002 Mar. 26 & $23: 37$ & $162 / 9$ & $-10,0,8 / 100$ & $12,22,30 / 122$ & 12.24 \\
\hline 2020 & 2002 Mar. 27 & 01:08 & $161 / 9$ & $-10,0,8 / 110$ & $12,22,30 / 132$ & 12.23 \\
\hline 2020 & 2002 Mar. 27 & 01:19 & $161 / 9$ & $-10,0,8 / 110$ & $12,22,30 / 132$ & 12.23 \\
\hline 2020 & 1996 Feb. 29 & $00: 11$ & 240 & $-4,12,50$ & & 12.41 \\
\hline 2550 & 2002 Mar. 25 & $23: 52$ & 2 & 10 & 20 & 10.33 \\
\hline 2550 & 2002 Mar. 26 & $00: 33$ & 2 & 10 & 20 & 10.33 \\
\hline 2550 & 2002 Mar. 26 & $01: 56$ & 2 & 10 & 20 & 10.33 \\
\hline 2550 & 2002 Mar. 26 & 02:07 & 2 & 10 & 20 & 10.33 \\
\hline 2550 & 2002 Mar. 26 & $23: 52$ & 2 & 10 & 20 & 10.33 \\
\hline 2550 & 2002 Mar. 27 & 00:03 & 2 & 10 & 20 & 10.33 \\
\hline 2550 & 2002 Mar. 27 & 01:32 & 2 & 10 & 20 & 10.33 \\
\hline 2550 & 2002 Mar. 27 & $01: 43$ & 2 & 10 & 20 & 10.33 \\
\hline 2550 & 1996 Feb. 29 & $01: 24$ & 3 & 5 & & 10.50 \\
\hline 3485 & 2002 Mar. 26 & 00:07 & 5 & 27,38 & 3,13 & 10.72 \\
\hline 3485 & 2002 Mar. 26 & $00: 46$ & 5 & 27,38 & 3,13 & 10.72 \\
\hline 3485 & 2002 Mar. 26 & $02: 21$ & 5 & 27,38 & 3,13 & 10.72 \\
\hline 3485 & 2002 Mar. 26 & $02: 25$ & 5 & 27,38 & 3,13 & 10.72 \\
\hline 3485 & 2002 Mar. 27 & $00: 16$ & 5 & 27,38 & 3,13 & 10.72 \\
\hline 3485 & 2002 Mar. 27 & $00: 20$ & 5 & 27,38 & 3,13 & 10.72 \\
\hline 3485 & 2002 Mar. 27 & $01: 57$ & 5 & 27,38 & 3,13 & 10.72 \\
\hline 3485 & 2002 Mar. 27 & 02:01 & 5 & 27,38 & 3,13 & 10.72 \\
\hline 3485 & 1996 Feb. 28 & 05:09 & 6 & 25,40 & & 10.80 \\
\hline 3685 & 2002 Mar. 26 & $00: 13$ & 1.3 & 6 & 4 & 10.14 \\
\hline 3685 & 2002 Mar. 26 & $00: 52$ & 1.3 & 6 & 4 & 10.14 \\
\hline 3685 & 2002 Mar. 26 & $02: 32$ & 1.3 & 6 & 4 & 10.14 \\
\hline 3685 & 2002 Mar. 26 & $02: 36$ & 1.3 & 6 & 4 & 10.14 \\
\hline 3685 & 2002 Mar. 27 & $00: 27$ & 1.3 & 6 & 4 & 10.14 \\
\hline 3685 & 2002 Mar. 27 & $00: 31$ & 1.3 & 6 & 4 & 10.14 \\
\hline 3685 & 2002 Mar. 27 & 02:08 & 1.3 & 6 & 4 & 10.14 \\
\hline 3685 & 2002 Mar. 27 & $02: 12$ & 1.3 & 6 & 4 & 10.14 \\
\hline 3685 & 1996 Feb. 29 & 03:30 & 3 & 0 & & 10.50 \\
\hline 4796 & 2002 Mar. 26 & $02: 44$ & 3 & $-20,-10$ & $-4,-14$ & 10.50 \\
\hline 4796 & 2002 Mar. 26 & $05: 18$ & 3 & $-20,-10$ & $-4,-14$ & 10.50 \\
\hline 4796 & 2002 Mar. 27 & $02: 19$ & 3 & $-20,-10$ & $-4,-14$ & 10.50 \\
\hline 4796 & 2002 Mar. 27 & $04: 51$ & 3 & $-20,-10$ & $-4,-14$ & 10.50 \\
\hline 4796 & 1996 Feb. 29 & $03: 43$ & 3 & $-8,-9$ & & 10.50 \\
\hline 4881 & 2002 Mar. 26 & $03: 50$ & 35 & 5 & -2 & 11.57 \\
\hline 4881 & 2002 Mar. 26 & $06: 39$ & 35 & 5 & -2 & 11.57 \\
\hline 4881 & 2002 Mar. 27 & $03: 24$ & 35 & 5 & -2 & 11.57 \\
\hline 4881 & 2002 Mar. 27 & $06: 11$ & 35 & 5 & -2 & 11.57 \\
\hline 4881 & 1996 Feb. 28 & $07: 40$ & 44 & -24 & & 11.67 \\
\hline 5367 & 2002 Mar. 26 & $06: 24$ & 4 & $-30,-25,-18$ & $-22,-17,-10$ & 10.63 \\
\hline 5367 & 2002 Mar. 26 & 08:05 & 4 & $-30,-25,-18$ & $-22,-17,-10$ & 10.63 \\
\hline 5367 & 2002 Mar. 26 & 08:52 & 4 & $-30,-25,-18$ & $-22,-17,-10$ & 10.63 \\
\hline 5367 & 2002 Mar. 27 & $05: 56$ & 4 & $-30,-25,-18$ & $-22,-17,-10$ & 10.63 \\
\hline 5367 & 2002 Mar. 27 & $07: 36$ & 4 & $-30,-25,-18$ & $-22,-17,-10$ & 10.63 \\
\hline 5367 & 2002 Mar. 27 & $08: 23$ & 4 & $-30,-25,-18$ & $-22,-17,-10$ & 10.63 \\
\hline 5367 & 2002 Mar. 27 & 09:36 & 4 & $-30,-25,-18$ & $-22,-17,-10$ & 10.63 \\
\hline 5367 & 1997 Sep. 15 & $23: 57$ & 4 & -10 & & 10.63 \\
\hline 6519 & 2002 Mar. 26 & $08: 20$ & 18 & $3,6,11,16$ & $-28,-25,-20,-15$ & 11.28 \\
\hline 6519 & 2002 Mar. 26 & 09:21 & 18 & $3,6,11,16$ & $-28,-25,-20,-15$ & 11.28 \\
\hline 6519 & 2002 Mar. 27 & $07: 51$ & 18 & $3,6,11,16$ & $-28,-25,-20,-15$ & 11.28 \\
\hline 6519 & 2002 Mar. 27 & $08: 51$ & 18 & $3,6,11,16$ & $-28,-25,-20,-15$ & 11.28 \\
\hline 6519 & 1996 Feb. 29 & $07: 39$ & 23 & 10 & & 11.39 \\
\hline 6556 & 2002 Mar. 26 & 09:08 & 19 & -27 & -25 & 11.30 \\
\hline 6556 & 2002 Mar. 26 & $08: 38$ & 19 & -27 & -25 & 11.30 \\
\hline 6556 & 2002 Mar. 26 & 09:22 & 19 & -27 & -25 & 11.30 \\
\hline 6556 & 1996 Feb. 29 & $09: 15$ & 22 & -27 & & 11.37 \\
\hline
\end{tabular}




\subsubsection{HR 6556 ( $\alpha$ Oph)}

HR 6556 is the nearest star of our sample with a distance of only 14.3 pc. It is thus surprising that HR 6556 has a very strong absorption feature (see Fig. 9) with the measured equivalent width corresponding to a column density of $\log N\left(\mathrm{Ca}^{+}\right)=$ $11.3 \mathrm{~cm}^{-2}$.

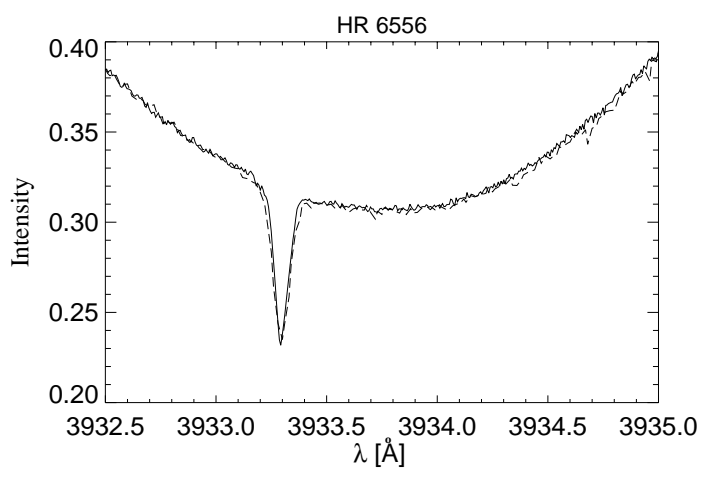

Fig. 9. Spectra of HR 6556 recorded on 2002 Mar. 26, UT 09:08 (solid line) and 1996 Feb. 29, UT 09:15 (dashed line). The extraordinary strong absorption feature of the nearest star in our sample is stable for years.

Interestingly, the other objects investigated in this paper do not show such a strong interstellar absorption although they are located at much larger distances. We note that Ferlet et al. (1986) report the detection of "at least three interstellar clouds" in the direction of $\alpha \mathrm{Aql}(d=5 \mathrm{pc})$. Crawford (2001) carried out a detailed study of HR 6556 and eight early-type stars located in the surrounding $15^{\circ}$ of the sky and found that the cloud responsible for the narrow CaII $\mathrm{K}$ absorption of HR 6556 "has a spatial extend of $\lesssim 1 \mathrm{pc}$ (and is probably very much less)". A possible explanation would be a very clumpy structure of our interstellar vicinity. Crawford points out that he cannot rule out the contribution of a possible CS component and suggest to search for temporal variability. However because we do not find variability in $\mathrm{CaII}$ as a direct evidence for CS gas, it is still not possible to conclude on the nature of this gas.

\section{Discussion and Conclusions}

In view of the stars analysed the question arises how to distinguish between circumstellar and interstellar absorptions. The search for both is extremely important since interstellar absorptions permit a study of the gas of the solar neighbourhood whereas studies of circumstellar absorptions help to study the gas phase of possible young planetary systems. Since the CaII $\mathrm{K}$ line serves as a diagnostic tool for the study of both CS and IS material we are confronted with the difficulty of disentangling an IS and a CS absorption (see also HHK and Crawford et al. 2002).

Unfortunately the case of $\beta$ Pictoris where the interpretation of the data is straightforward seems to be exceptional. The CaII $\mathrm{K}$ profile of $\beta$ Pictoris shows absorption components variable on short timescales. Even if the star was not a well-known
IRAS source and its circumstellar disk had not been coronographically resolved, the CS origin of HR 2020's variable absorptions would have been self-evident.

The data presented in Table 2 show that short-term variability is uncommon in our sample except for the well-known case of $\beta$ Pictoris. At first glance the most straightforward explanation for this result is that only $\beta$ Pictoris shows narrow absorption features of circumstellar material whereas the features detected in the CaII $\mathrm{K}$ lines of our other program stars seem to be of interstellar origin. A closer inspection of the data suggests, however, a different picture.

Ascribing the narrow absorptions - besides those of $\beta$ Pictoris - exclusively to interstellar material is in sharp contrast with the fact (shown by HHK) that the stellar parameter $v \sin i$ is the decisive factor for the occurrence of narrow absorption components and that these components occur preferably in stars with low gravities.

Our results presented in Table 2 show - besides the prototypical case of $\beta$ Pictoris - at least two new stars, namely HR 2550 and HR 3685, with variability in the narrow absorption features in the core of the CaII $\mathrm{K}$ line. From our present knowledge we cannot attribute the origin of the absorption component of HR 3685 to interstellar material. The case of HR 2550 is still unclear. Whereas for HR 2550 only the radial velocity with respect to the star of the detected feature changes, the situation for HR 3685 is more complex: not only the radial velocity with respect to the stellar profile has changed within 6 years, but also its shape. Despite the different spectral resolution of both datasets it is clearly visible that the 2002 feature (solid line in Fig. 3) is much narrower than the 1996 feature. This is a clear evidence for a circumstellar origin.

HR 4796 is another star where circumstellar material has been successfully imaged (Schneider et al. 1999). Therefore this star seems to be an ideal test object to check whether the FEB-scenario holds true for other stars as well. Indeed HR 4976 shows two narrow absorption features in CaII K. But in contrast to $\beta$ Pictoris they do not vary - even on long timescales.

We point out that even the detection of radial velocity variations of a narrow absorption does not necessarily prove its CS origin. HR 4881 and HR 5367 show absorption features with changing radial velocities with respect to the star. As remarked above the reason for this is the binarity of the stars. Thus multiple stars showing narrow absorption components can mimic variable (i.e. circumstellar) absorptions. These cases have to be scrutinized in more detail to distinguish between CS and IS origin.

\section{Summary}

The main results of this work are the following: (1) Short-term variations of CaII $\mathrm{K}$ absorption components are uncommon in our sample stars and could be found only for $\beta$ Pictoris. (2) Four stars of our sample show long-term variability. In two cases this is due to the binarity of the stars. (3) Stars with dust disks can have constant CaII $\mathrm{K}$ absorptions as can be seen from the case of HR 4796. 
Table 3. Overview of our results. In the second and third column we compile the results from our search for spectral variations based on the analysis of the CaII K line. RV indicates that we attribute the variation to the radial velocity of a binary system. In Cols. 4-7 the values of our column densities of the absorption features with their heliocentric velocities and those calculated from the LIC Model Column Density Calculator (Redfield \& Linsky 2002, 2000; Lallement \& Bertin 1992) are compared. $d_{\text {edge }}$ gives the distance to the edge of the LIC; for comparison, the distance to the sample stars is given in the last column.

\begin{tabular}{|c|c|c|c|c|c|c|c|c|}
\hline HR & $\begin{array}{l}\text { short-term } \\
\text { variation }\end{array}$ & $\begin{array}{l}\text { long-term } \\
\text { variation }\end{array}$ & $\begin{array}{r}\log N\left(\mathrm{Ca}^{+}\right) \\
\mathrm{cm}^{-2} \\
\text { this work }\end{array}$ & $\begin{array}{r}\log N\left(\mathrm{Ca}^{+}\right) \\
\mathrm{cm}^{-2} \\
\text { Colorado LIC }\end{array}$ & $\begin{array}{r}v_{\text {hel }} \\
\left(\mathrm{km} \mathrm{s}^{-1}\right) \\
\text { this work }\end{array}$ & $\begin{array}{r}v_{\text {hel }} \\
\left(\mathrm{km} \mathrm{s}^{-1}\right) \\
\text { Colorado LIC/G Cloud }\end{array}$ & $\begin{array}{r}d_{\text {edge }} \\
(\mathrm{pc})\end{array}$ & $\begin{array}{r}d \\
\text { (pc) }\end{array}$ \\
\hline 2020 & yes & yes & 12.26 & 9.59 & $12,22,30$ & $10.1 / 11.8$ & 0.55 & 19.3 \\
\hline 2550 & no & yes & 10.33 & 9.16 & 20 & $4.6 / 5.3$ & 0.21 & 30.3 \\
\hline 3485 & no & no & 10.72 & 8.97 & 3,13 & $2.6 / 2.4$ & 0.13 & 24.5 \\
\hline 3685 & no & yes & 10.14 & 8.83 & 4 & $-2.3 /-2.7$ & 0.1 & 34.1 \\
\hline 4796 & no & no & 10.50 & 8.59 & $-4,-14$ & $-11.9 /-14.8$ & 0.06 & 67.1 \\
\hline 4881 & no & yes (RV) & 11.57 & 8.55 & -2 & $-13.3 /-16.7$ & 0.05 & 60.2 \\
\hline 5367 & no & yes (RV) & 10.63 & 8.55 & $-22,-17,-10$ & $-19.1 /-22.6$ & 0.05 & 75.8 \\
\hline 6519 & no & no & 11.28 & 8.56 & $-28,-25,-20,-15$ & $-25.2 /-28.4$ & 0.05 & 130.7 \\
\hline 6556 & no & no & 11.30 & 8.70 & -25 & $-22.5 /-25.7$ & 0.07 & 14.3 \\
\hline
\end{tabular}

We emphasize that our program stars show a clear correspondence between narrow CaII $\mathrm{K}$ features and the stellar properties rapid rotation and low gravitiy (HRH, HHK). Furthermore, some of the stars are known to possess CS dust (cf. Table 1). Since reliable IS data are indispensable for comparison with heliocentric velocities of narrow absorptions and the study of the solar neighbourhood it is desirable to check this data for possible correlations with stellar properties like rotational velocities and $\log g$ values in order to exclude CS contributions.

Acknowledgements. We thank Inga Kamp and Hartmut Holweger for numerous helpful discussions. Furthermore, we would like to thank Ansgar Reiners for deriving benefit from his experience in handling CES-Data. This work was supported by the DLR under grant DLR 50 OR 0005.

\section{References}

Allen, C. W. 1973, Astrophysical Quantities, 3rd ed. (Athlone Press, London)

Bary, J. S., Weintraub, D. A., \& Kastner, J. H. 2002, ApJ, 576, L73

Berghöfer, T. W., Schmitt, J. H. M. M., \& Hünsch, M. 1999, A\&A, 342, L17

Beust, H., \& Morbidelli, A. 1996, Icarus, 120, 358

Beust, H., \& Morbidelli, A. 2000, Icarus, 143, 170

Beust, H., Vidal-Madjar, A., Ferlet, R., \& Lagrange-Henri, A. M. 1990, A\&A, 236, 202

Beust, H., Vidal-Madjar, A., Ferlet, R., \& Lagrange-Henri, A. M. 1991, A\&A, 241, 488

Beust, H., Lagrange, A.-M., Crawford, I. A., et al. 1998, A\&A, 338, 1015

Cheng, K. P., Bruhweiler, F. C., Kondo, Y., \& Grady, C. A. 1992, ApJ, 396, L83

Crawford, I. A., Craig, N., \& Welsh, B. Y. 1997, A\&A, 317, 889

Crawford, I. A. 2001, MNRAS, 327, 841

Crawford, I. A., Lallement, R., Price, R. J., et al. 2002, MNRAS, 337, 720

Dunkin, S. K., Barlow, M. J., \& Ryan, S. G. 1997, MNRAS, 290, 165 ESA 1997, The Hipparcos and Tycho Catalogues, ESA SP-1200
Ferlet, R., Lallement, R., \& Vidal-Madjar, A. 1986, A\&A, 163, 204 Ferlet, R., Vidal-Madjar, A., \& Hobbs, L. M. 1987, A\&A, 185, 267

Ferlet, R., Lagrange-Henri, A.-M., Beust, H., et al. 1993, A\&A, 267, 137

Grady, C. A., \& Silvis, J. M. S. 1993, ApJ, 402, L61

Hobbs, L. M., Vidal-Madjar, A., Ferlet, R., Albert, C. E., \& Gry, C. 1985, ApJ, 293, L29

Hoffleit, D., \& Warren Jr, W. H. 1991, The Bright Star Catalogue, 5th Revised Ed.

Holweger, H., \& Rentzsch-Holm, I. 1995, A\&A, 303, 819 (HRH)

Holweger, H., Hempel, M., \& Kamp, I. 1999, A\&A, 350, 603 (HHK)

Hünsch, M., Schmitt, J. H. M. M., Sterzik, M. F., \& Voges, W. 1999, A\&AS, 135, 319

Jura, M. 1991, ApJ, 383, L79

Kamp, I., \& Bertoldi, F. 2000, A\&A, 353, 276

Kamp, I., Hempel, M., \& Holweger, H. 2002, A\&A, 388, 978 (KHH)

Kamp, I., \& Paunzen, E. 2002, MNRAS, 335, L45

Kupka, F., Piskunov, N. E., Ryabchikova, T. A., Stempels, H. C., \& Weiss, W. W. 1999, A\&AS, 138, 119 (VALD-2)

Kurucz, R. L. 1992, Rev. Mex. Astron. Astrofis., 23, 181

Lacy, J. H., Richter, M. J., Greathouse, T. K., Jaffe, D. T., \& Zhu, Q. 2002, PASP, 114, 153

Lagrange-Henri, A. M., Ferlet, R., Vidal-Madjar, A., et al. 1990, A\&AS, 85, 1089

Lagrange-Henri, A. M., Gosset, E., Beust, H., Ferlet, R., \& VidalMadjar, A. 1992, A\&A, 264, 637

Lagrange, A. M., Vidal-Madjar, A., Deleuil, M., et al. 1995, A\&A, 296, 499

Lagrange, A.-M., Plazy, F., Beust, H., et al. 1996, A\&A, 310, 547

Lallement, R., \& Bertin, P. 1992, A\&A, 266, 479

Lecavelier des Etangs, A., Vidal-Madjar, A., Backman, D. E., et al. 1997a, A\&A, 321, L39

Lecavelier des Etangs, A., Deleuil, M., Vidal-Madjar, A., et al. 1997b, A\&A, 325, 228

Lecavelier des Etangs, A., Ferlet, R., \& Vidal-Madjar, A. 1997c, A\&A, 328, 602

Lecavelier des Etangs, A., Vidal-Madjar, A., Roberge, A., et al. 2001, Nature, 412, 706

Oudmaijer, R. D., van der Veen, W. E. C. J., Waters, L. B. F. M., et al. 1992, A\&AS, 96, 625

Petterson, O. K. L., \& Tobin, W. 1999, MNRAS, 304, 733 
Redfield, S., \& Linsky, J. L. 2002, ApJS, 139, 439

Redfield, S., \& Linsky, J. L. 2000, ApJ, 534, 825

Richter, M. J., Jaffe, D. T., Blake, G. A., \& Lacy, J. H. 2002, ApJ, 572, L161

Roberge, A., Feldman, P. D., Lecavelier des Etangs, A., et al. 2002, ApJ, 568, 343

Schneider, G., Smith, B. A., Becklin, E. E., et al. 1999, ApJ, 513, L127

Sfeir, D. M., Lallement, R., \& Welsh, B. Y. 1999, A\&A, 346, 785

Smith, B. A., \& Terrile, R. J. 1984, Science, 226, 1421

Stürenburg, S. 1993, A\&A, 277, 139

Telesco, C. M., Fisher, R. S., Piña, R. K., et al. 2000, ApJ, 530, 329
Thébault, P., \& Beust, H. 2001, A\&A, 376, 621

Thi, W. F., van Dishoeck, E. F., Blake, G. A., et al. 2001, ApJ, 561, 1074

Venn, K. A., \& Lambert, D. L. 1990, ApJ, 363, 234

Vidal-Madjar, A., Ferlet, R., Hobbs, L. M., Gry, C., \& Albert, C. E. 1986, A\&A, 167, 325

Vidal-Madjar, A., Lagrange-Henri, A.-M., Feldman, P. D., et al. 1994, A\&A, 290, 245

Waters, L. B. F. M., Cote, J., \& Geballe, T. R. 1988, A\&A, 203, 348

Welsh, B. Y., Craig, N., Crawford, I. A., \& Price, R. J. 1998, A\&A, 338,674 